\title{
A Dynamic Flight Model for Slocum Gliders and Implications for Turbulence Microstructure Measurements
}

\author{
LuCAS MERCKELBACH AND ANJA BERGER \\ Institute of Coastal Research, Helmholtz-Zentrum Geesthacht, Geesthacht, Germany \\ Gerd KRAHMANN AND MARCus DENGLER \\ GEOMAR Helmholtz Centre for Ocean Research Kiel, Kiel, Germany \\ JEFFREY R. CARPENTER \\ Institute of Coastal Research, Helmholtz-Zentrum Geesthacht, Geesthacht, Germany
}

(Manuscript received 20 September 2018, in final form 14 December 2018)

\begin{abstract}
The turbulent dissipation rate $\varepsilon$ is a key parameter to many oceanographic processes. Recently, gliders have been increasingly used as a carrier for microstructure sensors. Compared to conventional ship-based methods, glider-based microstructure observations allow for long-duration measurements under adverse weather conditions and at lower costs. The incident water velocity $U$ is an input parameter for the calculation of the dissipation rate. Since $U$ cannot be measured using the standard glider sensor setup, the parameter is normally computed from a steady-state glider flight model. As $\varepsilon$ scales with $U^{2}$ or $U^{4}$, depending on whether it is computed from temperature or shear microstructure, respectively, flight model errors can introduce a significant bias. This study is the first to use measurements of in situ glider flight, obtained with a profiling Doppler velocity log and an electromagnetic current meter, to test and calibrate a flight model, extended to include inertial terms. Compared to a previously suggested flight model, the calibrated model removes a bias of approximately $1 \mathrm{~cm} \mathrm{~s}^{-1}$ in the incident water velocity, which translates to roughly a factor of 1.2 in estimates of the dissipation rate. The results further indicate that $90 \%$ of the estimates of the dissipation rate from the calibrated model are within a factor of 1.1 and 1.2 for measurements derived from microstructure temperature sensors and shear probes, respectively. We further outline the range of applicability of the flight model.
\end{abstract}

\section{Introduction}

The dissipation rate of turbulent kinetic energy is a parameter that plays a key role in many physical and biogeochemical processes in oceans and coastal seas. However, direct oceanic measurements of turbulence are relatively scarce, as most observations stem from free-falling profilers, operated from seagoing vessels. The monetary and labor cost of taking such profiles is therefore substantial, and is most often limited to relatively calm conditions.

An emerging alternative to ship-based profiling is the use of ocean gliders, a class of buoyancy-driven autonomous underwater vehicles (Griffiths 2002, chapter 3; Rudnick 2016). Although gliders have been increasingly

\footnotetext{
Corresponding author: Lucas Merckelbach, lucas.merckelbach@ hzg.de
}

used over almost two decades, it is only recently that turbulence profilers have been mounted onto gliders (Wolk et al. 2009; Fer et al. 2014; Peterson and Fer 2014; Palmer et al. 2015; Schultze et al. 2017; St. Laurent and Merrifield 2017; Scheifele et al. 2018). The glider as a measurement platform has relatively low levels of vibration and mechanical noise, mainly because of the absence of a propeller. This makes gliders suitable for turbulence observations using shear probes. Using gliders as an alternative to oceangoing ships removes a substantial part of the human factor in the data collection process and therefore eases long-duration data collection while reducing costs, and it effectively removes any measurement constraints imposed by adverse weather conditions.

Despite the advantages of glider-based turbulence measurements, the major drawback of this setup is the uncertainty in the flight of the glider. A required parameter 
in the processing of microstructure shear and temperature measurements is the speed of flow past the sensors. The speed through water enters the processing raised to the fourth power when using airfoil shear probes, and to the second power when using microtemperature sensors (see section 6 a for details). For free-falling vertical profilers, this is usually determined from the rate of change of pressure. For glider-based measurements, this gives only the vertical speed of the glider, with a horizontal speed that must be either measured directly or modeled.

It is uncommon for a microstructure glider to be equipped with a device that directly measures (a horizontal component of) the speed through water, so often a flight model is used for the computation of turbulent dissipation (Fer et al. 2014; Peterson and Fer 2014; Palmer et al. 2015; Schultze et al. 2017; St. Laurent and Merrifield 2017; Scheifele et al. 2018).

The most commonly used flight model is that of Merckelbach et al. (2010), who assume a steady-state balance between buoyancy, drag, and lift, and use the measured pitch angle and buoyancy change achieved by the buoyancy engine to compute the speed through water (Fer et al. 2014; Peterson and Fer 2014; Palmer et al. 2015; Schultze et al. 2017; Scheifele et al. 2018). The numerical evaluation of these forces requires values to be attributed to a number of coefficients, such as the glider density, compressibility, drag, and lift coefficients. Merckelbach et al. (2010) show that the glider density, compressibility, and drag coefficients can be determined from standard glider sensors; however, they note that it is not possible to simultaneously determine the lift coefficient without a direct measurement of the horizontal component of the glider speed. ${ }^{1}$ The model therefore relies on tabulated coefficients from aerodynamic studies of bodies of similar shape in its specification of lift coefficients. To date, no study has been published where the glider flight model by Merckelbach et al. (2010) is calibrated and compared with direct measurements of the glider velocity through water.

In summary, glider-based turbulence microstructure measurements represent new possibilities for sampling ocean turbulence, but they suffer from uncertainties in glider flight models, which are particularly sensitive, and as yet untested. In this work a Teledyne Webb Research Slocum Electric Shallow glider has been

\footnotetext{
${ }^{1}$ Depth-averaged velocities computed using surface GPS data, as attempted by Merckelbach et al. (2010), would provide such a measurement. However, for calibrating the lift coefficient, water velocities along the track of the glider must be known with a bias of less than approximately $1 \mathrm{~cm} \mathrm{~s}^{-1}$, making this method unreliable.
}

fitted with a Teledyne RD Instruments (RDI) Explorer Doppler velocity $\log$ (DVL). This device primarily measures the horizontal and vertical components of the glider velocity with respect to the seabed (bottom track), provided the seabed is within the acoustic range of about $80 \mathrm{~m}$. A profiling mode also allows the measurement of the glider velocity relative to the water at some distance below the glider (about 5-15 m). In addition, we also utilize data collected from an electromagnetic current meter mounted inside a microstructure package to assess glider flight characteristics. Using these data we calibrate and extend the glider flight model of Merckelbach et al. (2010) based on direct measurements of glider flight, as well as examine the implications for the accuracy of turbulent dissipation rate estimates as measured with glider-mounted microstructure sensors.

\section{Background: Steady-state glider flight model}

Key to the work presented herein is a steady-state planar glider flight model developed by Merckelbach et al. (2010) in order to obtain vertical water velocities from glider observations. The model is based on a horizontal $(x)$ and vertical $(z)$ force balance, in which the acceleration terms are neglected, given by ${ }^{2}$

$$
\begin{aligned}
& 0=\sin (\theta+\alpha) F_{L}-\cos (\theta+\alpha) F_{D} \\
& 0=F_{B}-F_{g}-\cos (\theta+\alpha) F_{L}-\sin (\theta+\alpha) F_{D},
\end{aligned}
$$

where the pitch angle $\theta$ and the angle of attack $\alpha$ are defined in Fig. 1. The forces that act on the glider are due to buoyancy $F_{B}$, gravity $F_{g}$, lift $F_{L}$, and drag $F_{D}$ :

$$
\begin{aligned}
F_{g} & =m_{g} g, \\
F_{B} & =g \rho\left\{V_{g}\left[1-\varepsilon_{c} P+\alpha_{T}\left(T-T_{0}\right)\right]+\Delta V_{\mathrm{bp}}\right\}, \\
F_{D} & =\frac{1}{2} \rho S U^{2}\left(C_{D_{0}}+\alpha^{2} C_{D_{1}}\right), \\
F_{L} & =\frac{1}{2} \rho S U^{2} C_{L}(\alpha), \\
C_{L}(\alpha) & =a \alpha,
\end{aligned}
$$

where $m_{g}$ is the mass of the glider, $g$ is the acceleration due to gravity, $\rho$ is the in situ density, $V_{g}$ is the volume of the glider at atmospheric pressure, $\varepsilon_{c}$ is the coefficient of

\footnotetext{
${ }^{2}$ This system of equations is identical to the equations given by Merckelbach et al. (2010), if their equations are corrected for an unfortunate sign error.
} 


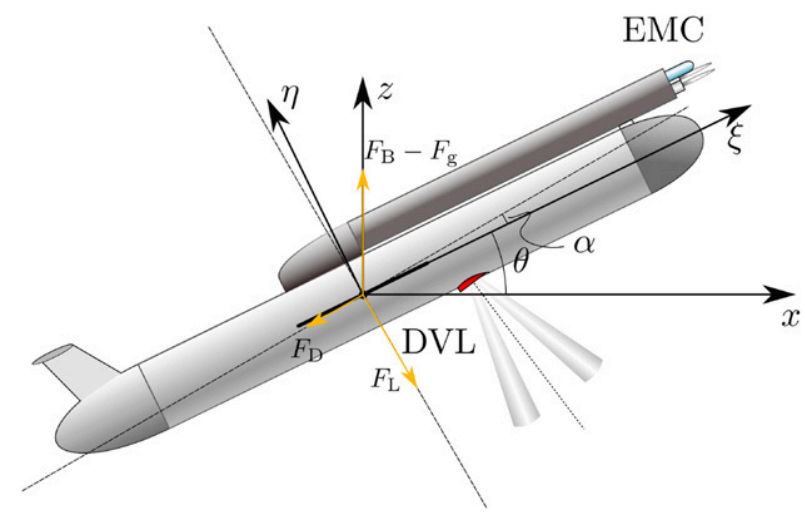

FIG. 1. Schematic representation of a glider, defining an orthogonal coordinate system with $x$ and $z$ axes, and an orthogonal glider referenced coordinate system $(\xi, \eta)$. The latter originates from the former after a rotation of the pitch angle. The glider path (dashed line) makes a small angle, equal to the angle of attack, with the glider's principal axis, which coincides with the $\xi$ axis. The forces resulting drag, lift, and net buoyancy are drawn by the yellow vectors. The positions of the EMC sensor and the DVL are in blue and red, respectively. The DVL is mounted such that the principal axis of the DVL (dashed) makes an $11^{\circ}$ angle with the $\eta$ axis.

compressibility, $\alpha_{T}$ is the thermal expansion coefficient of the glider, $\Delta V_{\mathrm{bp}}$ is the volume change achieved by the buoyancy engine, $S$ is the total surface area of the wings, $U$ is the magnitude of the glider speed through water, and $C_{D_{0}}$ and $C_{D_{1}}$ are the parasite and induced drag coefficients, respectively. For small $\alpha$, the lift coefficient $C_{L}$ is assumed to be linear in the angle of attack, proportional to the lift angle coefficient $a$.

An expression for $U$ can be obtained by either eliminating $F_{D}$ or $F_{L}$ from (1) and (2), and substituting from (5) and (6), respectively, relating $U$ to either drag coefficients or lift angle coefficients. Expressed in drag coefficients, we get

$$
\sin (\theta+\alpha)\left(F_{B}-F_{g}\right)-\frac{1}{2} \rho S U^{2}\left(C_{D_{0}}+C_{D_{1}} \alpha^{2}\right)=0 .
$$

In addition, an expression for the angle of attack is found by combining (1), and (5)-(7), yielding

$$
\alpha=\frac{C_{D_{0}}+\alpha^{2} C_{D_{1}}}{a \tan (\theta+\alpha)} .
$$

Equations (8) and (9) provide a model to compute the steady-state flight condition at any time, given measurements of the buoyancy drive, pitch angle, and in situ density, and a set of model coefficients for drag, lift, compressibility, and thermal expansion (if applicable).

Merckelbach et al. (2010) determine model coefficients by minimizing a cost function that is mathematically identical to

$$
R_{0}=\frac{1}{N} \sum_{i=0}^{N-1}\left\{U[i] \sin (\theta[i]+\alpha[i])+\frac{d h}{d t}[i]\right\}^{2} .
$$

Herein, the depth rate $d h / d t$ and pitch $\theta$ are observations with index $i$ from a total of $N$ values, and $U$ and $\alpha$ are the corresponding model results using (8) and (9), respectively. It can be shown, however, that the cost function does not yield unique values if both the parasite drag and the lift angle coefficient are present in the minimization parameter space. Although the model may be optimized for the vertical velocity component, the horizontal velocity component, and therefore the speed through water, depends on the actual value of the lift angle coefficient. This means that depth-rate observations alone are not sufficient to calibrate a glider flight model that also computes accurately the glider speed through water. Consequently, additional measurements of a nonvertical glider velocity component are required and are presented in section 4.

\section{Dynamic glider flight model}

Although the steady-state glider flight assumption seems reasonable for most practical situations, a dynamic nonsteady-state glider flight model may provide a better estimate of the glider speed for rapidly changing conditions, for example, when strong density gradients are present or around dive-to-climb turning points. Requiring little additional effort, such a dynamic flight model can be obtained by (re)inserting the acceleration terms into the steady-state model. Besides the glider mass accounting for its inertia, the so-called added mass needs to be considered. Added mass terms arise from the fact that if a submerged body accelerates, not only does the body's mass oppose the acceleration, but also the flow around the body changes. From an energy principle, it then follows that the body does work on the ambient water mass and additional forces act on the submerged body (Imlay 1961). These additional forces can be conveniently written as a $6 \times 6$ mass matrix, multiplied by a vector composed of three linear and three angular acceleration components (Newman 1977).

For planar flight with negligible rotational accelerations, which are typical for glider flight, the inertial forces to be inserted into the dimensional steady-state model can be simplified to $\mathbf{M}[d u / d t \quad d w / d t]^{\mathrm{T}}$. Herein $u$ and $w$ are the horizontal and vertical glider velocity components in a georeferenced coordinate system, respectively; and $\mathbf{M}$ is a $2 \times 2$ matrix, composed of the glider mass on the diagonal, $m_{\mathrm{g}} \mathbf{I}$, where $\mathbf{I}$ is the identity matrix, and a $2 \times 2$ matrix $\mathbf{m}$, representing the added mass terms. When expressed in the orthogonal glider referenced coordinate 
system, $(\xi, \eta)$ (see Fig. 1), with its axes (from the glider's perspective) pointing forward and upward, the added $\mathbf{m}$ for a glider-shaped object can adequately be described by a diagonal matrix, $\operatorname{diag}\left(m_{11}, m_{22}\right)$, where $m_{11}$ and $m_{22}$ are

$$
\mathbf{M}=m_{g} \mathbf{I}+\left[\begin{array}{cc}
m_{11} \cos ^{2}(\theta)+m_{22} \sin ^{2}(\theta) & \left(m_{11}-m_{22}\right) \cos (\theta) \sin (\theta) \\
\left(m_{11}-m_{22}\right) \cos (\theta) \sin (\theta) & m_{g}+m_{22} \cos ^{2}(\theta)+m_{11} \sin ^{2}(\theta)
\end{array}\right]
$$

Based on the expressions given in Imlay (1961) for a finned prolate spheroid-shaped glider with a length of $2 \mathrm{~m}$, the numerical values of the added mass terms are estimated at $m_{11}=0.2 m_{g}$ and $m_{22}=0.92 m_{g}$.

With this result the dynamic flight model can be written as [see (1), (2)]

$\mathbf{M} \frac{d}{d t}\left[\begin{array}{c}u \\ w\end{array}\right]=\left[\begin{array}{c}\sin (\theta+\alpha) F_{L}-\cos (\theta+\alpha) F_{D} \\ F_{B}-F_{g}-\cos (\theta+\alpha) F_{L}-\sin (\theta+\alpha) F_{D}\end{array}\right]$.

To integrate this initial value problem using a classical Runge-Kutta (RK4) explicit method, we specify $u=w=0$ as initial conditions and when the glider is at the surface. Furthermore, the incident flow velocity is set equal to $U=\sqrt{u^{2}+w^{2}}$. In fact, this is true only if the water column behaves as a steady-state flow without shear. However, under most conditions the oceanic shear is small so that the errors of the estimated lift and drag forces can be assumed small too. We also note that transient effects caused by changing flow conditions on the drag and lift forces generated are not accounted for. Both the steady-state and dynamic models are implemented in Python 3. The source code, documentation, and examples are available at a public repository under the MIT software license (Merckelbach 2018a,b).

\section{Experimental data}

\section{a. Instrumentation}

The glider COMET is a Teledyne Webb Research G2 shallow electric glider that is equipped with a Sea-Bird Scientific glider payload CTD, a Rockland Scientific MicroRider, and a profiling Teledyne RDI $600-\mathrm{kHz}$ phased array Explorer DVL. The CTD received a firmware update allowing it to sample at $1 \mathrm{~Hz}$, rather than the default $0.5 \mathrm{~Hz}$. A thermal lag correction algorithm, similar to that described by Garau et al. (2011), was applied to correct the measured conductivity within the conductivity cell. The MicroRider sampled pressure and pitch at $64 \mathrm{~Hz}$.

The DVL mounted on glider COMET measured velocities acoustically along four beams in a Janus configuration at a $30^{\circ}$ angle. The device was mounted in a the dominant added mass components (Imlay 1961; Newman 1977). Expressed in the $(x, y)$ coordinate system, the transformed $\mathbf{m}$ is not necessarily diagonal, and the inertia matrix becomes

separate hull section, placed in front of the science bay (Fig. 1). Since the DVL is primarily designed to measure bottom-track velocities, it was installed downward looking and mounted with a forward pitch angle of $11^{\circ}$. The mounting angle ensures that if the glider is at a nominal dive angle of $26^{\circ}$, then the principal axis of the tetrahedron defined by three of its beams is aligned with the vertical ordinate.

Besides measuring bottom-track velocities, the DVL was configured (by means of a firmware upgrade) to collect three-dimensional current profiles, like a classical acoustic Doppler current profiler (ADCP). The DVL was set up to continuously record ensembles, each consisting of 10 profile pings with a bin size of $2 \mathrm{~m}$ and two bottom-track pings. With a typical ping rate of $7 \mathrm{~Hz}$ (Teledyne RD Instruments 2017), the measurement time of an ensemble amounted to up to $1 \mathrm{~s}$, during which the vertical distance traveled by the glider was about $10-15 \mathrm{~cm}$. The realized sample rate of the ensembles was between once per 4 and $3 \mathrm{~s}$, indicating a significant amount of time required to process and store the ensemble data.

According to the DVL's datasheet, the standard deviations for single-bottom-track and velocity profile pings are 1.0 and $4.7 \mathrm{~cm} \mathrm{~s}^{-1}$, respectively. For the configuration used, the standard deviation of the profile velocity relative to the seabed, computed from an ensemble, amounts to $1.7 \mathrm{~cm} \mathrm{~s}^{-1}$, assuming that all pings can be treated as independent variables. As this is calculated for ideal conditions, we use a more conservative value, estimated at $\sigma=2.5 \mathrm{~cm} \mathrm{~s}^{-1}$, allowing for additional uncertainty resulting from vertical shear, horizontal heterogeneity of the flow, and pitch, heading, and roll readings.

The DLV measurements are georeferenced using the pressure measured by the glider, after correcting for a small delay of about $3 \mathrm{~s}$ in DVL measurements. The time delay was computed for each profile by matching the glider and DVL time stamps of the pitch, a parameter that is measured by the glider's attitude sensor and fed into the DVL.

Raw DVL data were subjected to a number of qualitychecking algorithms to mask low-quality data as well as data correction algorithms. Following Todd et al. (2017), a pipeline of operations was set up to correct the speed of 

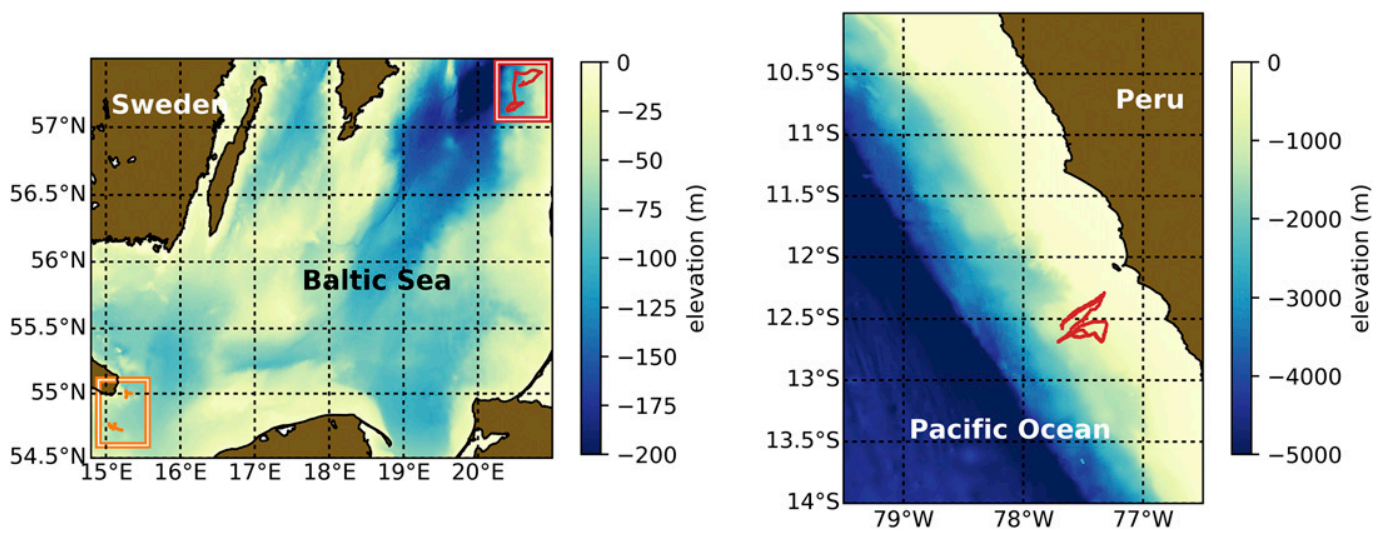

FIG. 2. Maps of where glider missions were carried out. (left) The Baltic Sea, where two missions were carried out with glider COMET, yielding dataset I (2016; inside orange box) and dataset II (2017; inside red box). (right) The coastal shelf of Peru (Pacific Ocean), yielding dataset III (2017).

sound using the salinity measured by the glider; to correct for offsets in roll and pitch; to mask relative water velocities and bottom-track velocities that exceed $0.75 \mathrm{~m} \mathrm{~s}^{-1}$; to mask velocities for which the signal-to-noise ratio (SNR) is smaller than 3 , where $\mathrm{SNR}=10^{\left(S_{\mathrm{dB}}-N_{\mathrm{dB}}\right) / 10}$ with $S_{\mathrm{dB}}$ and $N_{\mathrm{dB}}$ as the signal and noise levels (dB), respectively; and, finally, to mask velocities, the signal levels of which exceed $75 \mathrm{~dB}$. It is noted that in this work, the SNR threshold was set more permissively to 3 , rather than 20 as used in Todd et al. (2017).

To compute eastward, northward, and upward velocity components, the DVL uses heading, pitch, and roll angles that are reported by the glider. It was found that the difference between the upward bottom-track velocity and the depth rate was positively biased for upcasts and negatively biased for downcasts. It turned out that the tilt sensor had leaked a small amount of electrolyte fluid, so pitch and roll angles were reported larger than they were in reality. The associated error in the pitch and roll angles is proportional to their real values (D. Pheifer 2018, personal communication). Matching upward bottomtrack velocity and depth rate yielded a scaling factor of 0.83 . Prior to the step to correct the pitch and roll offsets in the processing pipeline, the DVL velocities were recomputed using scaled pitch and roll angles.

The glider IFM03 is a Teledyne Webb Research G1 deep glider (short version) equipped with an unpumped Sea-Bird CTD and a Rockland Scientific MicroRider (similar to the one mounted on top of the glider COMET). Added to this MicroRider (by Rockland Scientific) was an electromagnetic current (EMC) sensor (AEM1-G, by JFE Advantech Co., Ltd). CTD data were recorded at a sample rate of $1 \mathrm{~Hz}$ and were corrected for thermal lag effects following Garau et al. (2011). The relevant MicroRider data (pitch, pressure, and EMC velocity) were logged at a rate of $64 \mathrm{~Hz}$.
The EMC sensor measures the axial speed of the glider through the water. The sensor was calibrated in a water tank by the manufacturer JFE Advantech. This was done by towing the EMC sensor mounted to a MicroRider hull at different speeds through the tank. Although the EMC sensor is sampled at $64 \mathrm{~Hz}$, the sensor itself has a measurement frequency of $15 \mathrm{~Hz}$. During the postprocessing the velocity measurements were averaged to yield a time series with a frequency of $1 \mathrm{~Hz}$, with each sample being the average of about 10-15 individual measurements. The accuracy of the velocity measurements claimed by Rockland Scientific (2017) is $0.5 \mathrm{~cm} \mathrm{~s}^{-1}$ or $2 \%$ of the readings, without stating the measurement bandwidth. It is therefore not clear how much averaging of raw data samples is required to obtain this accuracy. We assume that the uncertainty in the EMC readings is $1 \mathrm{~cm} \mathrm{~s}^{-1}$ or better for the velocity data averaged to $1-\mathrm{Hz}$ time series.

\section{b. Datasets}

For the analysis of the glider flight model three datasets were selected (see Fig. 2 and Table 1 for a summary). Dataset I was selected because the data of glider COMET include water velocity measurements measured with a DVL. Importantly, the water depth was shallow enough ( $\approx 60 \mathrm{~m})$ for the DVL to record bottom-track velocities during the glider's downcasts, which is essential to compute relative glider velocities (section 4c). Because of the presence of a strong halocline, the dives were limited to depths of $40 \mathrm{~m}$. The glider was flown with pitch angles set to $\pm 26^{\circ}$.

Dataset III was selected because IFM03 carried a MicroRider that was retrofitted with an EMC, the measurement principle of which is entirely different from the DVL. In contrast to dataset I, this dataset also contains reliable velocity measurements on the upcasts, as well as during the transitions from dive to climb. 
TABLE 1. Dates and regions of the datasets used.

\begin{tabular}{clclcc}
\hline \hline Dataset & Glider & Velocity sensor & \multicolumn{1}{c}{ Region } & \multicolumn{1}{c}{ Start } & End \\
\hline I & COMET & DVL & Baltic Sea (southwest) & 20 Jun 2016 & 26 Jun 2016 \\
II & COMET & - & Baltic Sea (central) & 19 Oct 2017 & 28 Oct 2017 \\
III & IFM03 & EMC & Peru & 29 Apr 2017 & 23 May 2017 \\
\hline
\end{tabular}

This glider was programmed to dive to $800 \mathrm{~m}$, or when limited by topography to $15 \mathrm{~m}$ above the seabed. From this dataset we selected a sequence of profiles where the glider dives to the full $800 \mathrm{~m}$. The glider was flown with fixed battery positions, resulting in typical downcast and upcast pitch angles of $32^{\circ}$ and $28^{\circ}$, respectively.

Dataset II used the same glider setup as dataset I; however, the glider was flown in water depths that were too large to obtain bottom-track velocities for most of the profiles. Hence, the DVL data from this dataset are not used. This dataset is still of interest, because the day prior to recovery was used to fly the glider for short periods at two different pitch angles than the standard $26^{\circ}$-namely, $\pm 20^{\circ}$ and $\pm 30^{\circ}$-in order to quantify the effects of the induced drag.

\section{c. Relative glider velocities from DVL measurements}

The velocity profile data, configured to be outputted by the DVL as eastward, northward, and upward velocity components, represent the water velocity relative to the glider. The first bin is found at $2.92 \mathrm{~m}$ from the transducer. However, the data from the first two bins often show a signal that is distinctively different from the other bins and therefore the first two bins are excluded from the analysis to follow. The echoes from bins positioned farther away than about $15 \mathrm{~m}$ were often weak, not yielding a signal with a sufficiently high signal-tonoise ratio. As a result, the typical range for which meaningful data were obtained is some $7-15 \mathrm{~m}$ away from the glider. In the presence of significant vertical shear, the relative velocity of the glider measured by the DVL from a particular depth may not represent the actual relative velocity at the depth of the glider. To improve the estimates of the relative water velocity at the glider's position, we used the following approach.

Consider a glider collecting data using a downwardlooking DVL while diving (Fig. 3). When a profile ensemble is collected, water velocities relative to the glider are measured at a distance of about $10 \mathrm{~m}$ below the glider and turned into absolute water velocities by accounting for the absolute glider velocity using the bottom-track velocity. Some hundreds of seconds later, assuming a nominal depth rate of $10 \mathrm{~cm} \mathrm{~s}^{-1}$, the glider reaches a depth at which it previously collected velocity data. On the assumption that vertical changes in currents are much greater than horizontal changes, we estimate the water velocity at the current glider's depth from previously collected profile ensembles and then compute the relative glider velocity by subtracting the glider absolute velocity (bottom-track velocity) at this depth.

The drawback of this method is that in estimating the water velocity components at the glider's position, an average is constructed from profile ensembles taken 100-200 s earlier. No tendencies, or "future" profile ensemble data are taken into account. As an alternative, we also implemented a simple Kalman filter. A Kalman filter operates by propagating the mean and covariance of a state using a dynamic model, in an optimal way, given a time series of observations of the process (see, e.g., Anderson and Moore 2005; Simon 2006). As for a dynamic model we choose a simple one: the acceleration of a current component (eastward, northward, or upward) at a given depth is constant, with a model uncertainty of $\sigma^{2}=1 \times 10^{-16} \mathrm{~m}^{2} \mathrm{~s}^{-4}$ to reflect the fact that the model is only approximate in describing the real system. The state vector consists of a velocity component at a given depth and the corresponding acceleration. This filter is run for each component and depth bin separately, using measurements of the water velocity when they become available. The variance of the measurement noise is estimated at $\sigma^{2}=0.025^{2} \mathrm{~m}^{2} \mathrm{~s}^{-2}$; see also section 4 a. The filter is implemented as a forward-backward smoother, or a socalled RTS filter after Rauch, Tung, and Striebel who presented this filter in 1965; see Simon (2006). This filter is first run forward and then backward in time, making maximal use of available data.

In comparison with the Kalman filter, the averaging method is much simpler to implement and is also computationally considerably more efficient. The Kalman filter method, however, produces smoother, less noisy data and has been used in the results reported in this work.

\section{d. Incident water velocity from EMC measurements}

The one-dimensional EMC sensor measures the velocity component along the principal axis of the glider (the $\xi$ axis; see Fig. 1). Therefore, the incident water velocity derived from this sensor $U_{\mathrm{EMC}}$ relates to the actual measured velocity $\hat{U}_{\text {EMC }}$, as

$$
U_{\mathrm{EMC}}=\frac{\hat{U}_{\mathrm{EMC}}}{\cos (\alpha)} .
$$



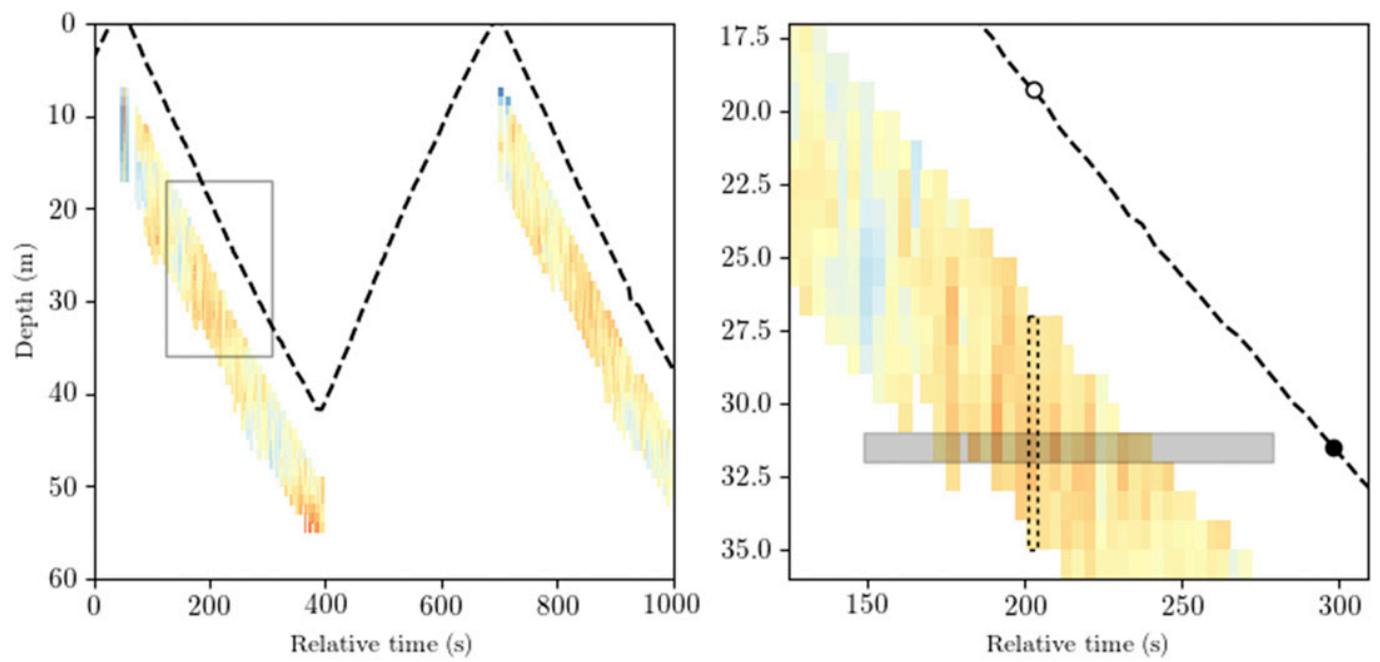

FIG. 3. Construction of water current profiles, expressed in a geographic Cartesian reference frame, as measured by a profiling glider. The dashed line represents the glider's path. (left) Current profiles are measured during the downcast only. (right) A zoomed-in view of the rectangle in the left panel. At $t=200 \mathrm{~s}$, the glider (indicated by the open circle) collects an absolute velocity profile, of which usable data are delineated by the dashed rectangle. Some hundreds of seconds later, the absolute velocity at the depth of the glider (solid circle) can be estimated from previous measurements (shaded rectangle). Subtracting the bottom-track velocity from the thus estimated absolute velocity yields the relative velocity.

Since $\alpha$ is not measured, values computed from the steady-state model, for example, can be used instead. These are generally small, so $\cos (\alpha)=1+\mathscr{O}\left(\alpha^{2}\right)$. However, it is noted that as a result of local shear, the angle of attack may not always be small, leading to (13) being a lower-bound estimate of the actual incident velocity.

During the processing of the EMC data, it was found that the vertical water velocity computed from $U_{\mathrm{EMC}}$ and the glider's pitch angle was consistently larger in magnitude than the measured depth rate. In contrast to the glider COMET, we have confidence in the pitch angles reported by glider IFM03, as they were nearly identical to the pitch angles reported by its MicroRider sensor. Therefore, we applied a scaling factor to the velocities reported by the EMC so that the difference between the vertical velocity component and the depth rate vanishes. Using the angle of attack estimated from the steady-state model (with lift angle and induced drag settings found for glider COMET; see next section), the factor was found to be equal to 0.93. A similar scaling factor was found by the MircoRider's manufacturer during tests with a SeaExplorer glider with a built-in MicroRider fitted with an EMC sensor, and an additionally mounted ADCP (R. Lueck 2018, personal communication).

\section{Glider flight model calibration and results}

It is not possible to find optimal choices for both $C_{D_{0}}$ and $a$ when using only the depth-rate measurement as a model constraint; an additional velocity measurement with a significant orthogonal (horizontal) component is required. In this section we use measurements of the incident water velocity as an additional model constraint to calibrate for the lift angle coefficient. This is done first for the DVL measurements and then for the EMC measurements.

Numerical values of drag and lift coefficients have a meaning only, if referenced to a known surface area $S$ [see also (5) and (6)]. In this study we follow the conventions used in aerodynamics and use the surface area of the wings as reference area, giving $S=0.1 \mathrm{~m}^{2}$. Another choice for $S$ could be the frontal area. To express drag and lift coefficients, referenced to the frontal area, the numerical values found in this study are to be multiplied by the ratio of wing area to frontal area.

In the subsections below, the value for $C_{D_{1}}$ used by the flight models is preset to $10.5 \mathrm{rad}^{-2}$, in anticipation of the result presented at the end of this section, where we also estimate the optimal value of $C_{D_{1}}$.

\section{a. Lift angle coefficient}

A simple approach is taken to estimate the optimal value of $a$. To that end, an additional cost function $R_{1}$ is defined as

$$
R_{1}=\frac{1}{N} \sum_{i=0}^{N-1}\left(U[i]-U_{\mathrm{DVL}}[i]\right)^{2}
$$

where $U_{\mathrm{DVL}}[i]$ is the incident water velocity derived from the DVL measurements with $i$. The cost function 


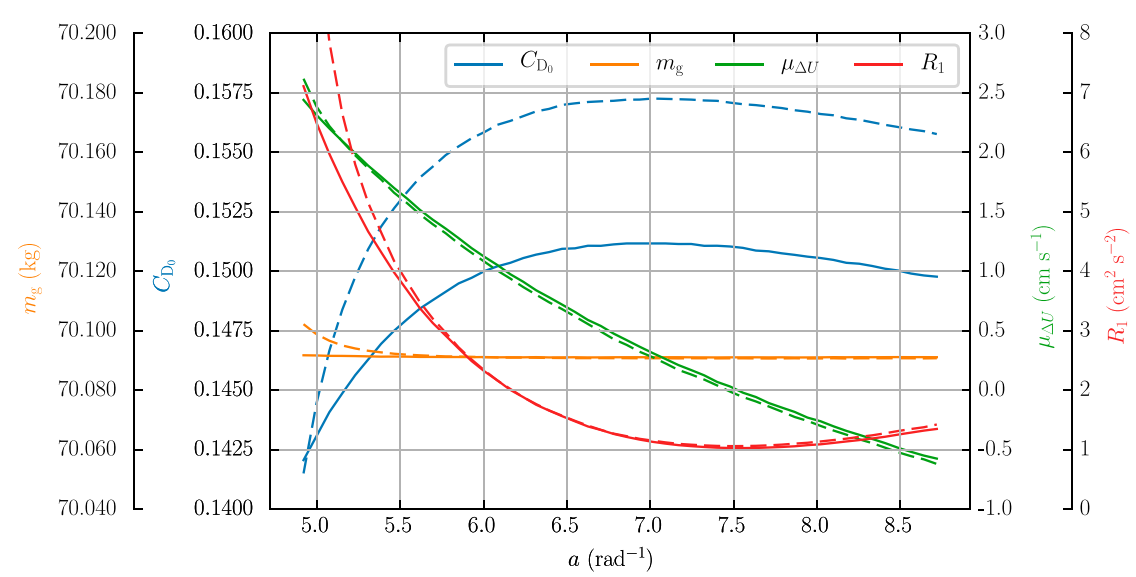

FIG. 4. Dependency of $m_{g}$ and $C_{D_{0}}$ on $a$ (left ordinate axes), and corresponding mean and standard deviations of the difference between the modeled and measured (DVL) incident velocity as well as $R_{1}$ (right ordinate axes). Results are shown for the steady-state model (solid lines) and the dynamic model (dashed lines). The mean of the incident velocity difference is denoted by $\mu_{\Delta U}$.

$R_{0}$ [(10)] is minimized for the parameter space $\left\{C_{D_{0}}, m_{g}\right\}$, for a range of preset values of $a$. Then, for each triplet $\left(C_{D_{0}}, m_{g}, a\right)$, the cost function $R_{1}$ is evaluated.

Figure 4 summarizes the results of these successive minimization steps, using the steady-state model (solid lines) and the dynamic model (dashed lines) applied to data from a subinterval of $4 \mathrm{~h}$ of data collected on 23 June 2017. The figure shows the optimal values for the parasite drag coefficient and the mass (by minimizing $R_{0}$ ) for a range of preset values of $a$. It is seen that the mass is independent of the value of the lift angle coefficient but that the drag coefficient is not. Moreover, the steady-state model estimates lower values for $C_{D_{0}}$ than the dynamic model does, the explanation of which is left for section 5c. The optimal value for $a$ (for which $R_{1}$ is minimal) is found to be $a \approx 7.4 \mathrm{rad}^{-1}$, for both models, where also the mean difference between the modeled and observed incident velocity is approximately zero.

We can now repeat the procedure to determine the glider flight model parameters $C_{D_{0}}, m_{g}$, and $a$, but using the EMC-derived incident velocity instead as the required nonvertical velocity component. The results are shown in Fig. 5 and are found to be in line with the results obtained from the DVL data (cf. Fig. 4). The data show a similar relationship between the optimized lift and drag coefficients, and the mass appears to be independent of the lift angle coefficient. For this glider (IFM03) an unbiased difference between measured and modeled incident velocities is found for $a=7.5 \mathrm{rad}^{-1}$, which is slightly higher than found for glider COMET.

In contrast to the DVL, the EMC provides continuous velocity data on both the up- and downcasts, so that $R_{0}$ can be modified to include a nonvertical velocity component, yielding

$$
\begin{aligned}
R_{2}= & \frac{1}{N} \sum_{i=0}^{N-1} \kappa\left\{U[i] \sin (\theta[i]+\alpha[i])+\frac{d h}{d t}[i]\right\}^{2} \\
& +(1-\kappa)\left(U[i]-U^{\mathrm{EMC}}[i]\right)^{2},
\end{aligned}
$$

where $\kappa$ is a weighting coefficient, set to $\kappa=1 / 2$, giving both velocity components equal importance assuming that the accuracy of their measurements is similar. The additional constraint allows for minimizing $R_{2}$ for the parameter triplet $C_{D_{0}}, m_{g}$, and $a$ simultaneously, yielding $C_{D_{0}}=0.136, m_{g}=59.454 \mathrm{~kg}$, and $a=7.7 \mathrm{rad}^{-1}$, indicated by the cross symbols in Fig. 5 .

The values for the lift angle coefficient, found for the gliders COMET (DVL) and IFM03 (EMC), are only slightly different. Figures 4 and 5 show, however, that a variation in $a$ of $1 \mathrm{rad}^{-1}$ would lead to a bias in the incident velocity of approximately 6 and $3 \mathrm{~mm} \mathrm{~s}^{-1}$ for gliders COMET and IFM03, respectively. Given the uncertainties in the velocity measurements, we consider these findings to be consistent.

\section{b. Induced drag coefficient}

The induced drag coefficient $C_{D_{1}}$ is another shape parameter, the setting of which may influence the results after calibrating the model for $m_{g}, C_{D_{0}}$, and $a$. From (9) it follows that the effect of the induced drag can be absorbed into the parasite drag coefficient if the glider is flown with pitch angles that are similar in magnitude for the up- and downcasts and (near) constant over time. In most cases this is how gliders are operated, and this second-order effect has little consequence on the model 


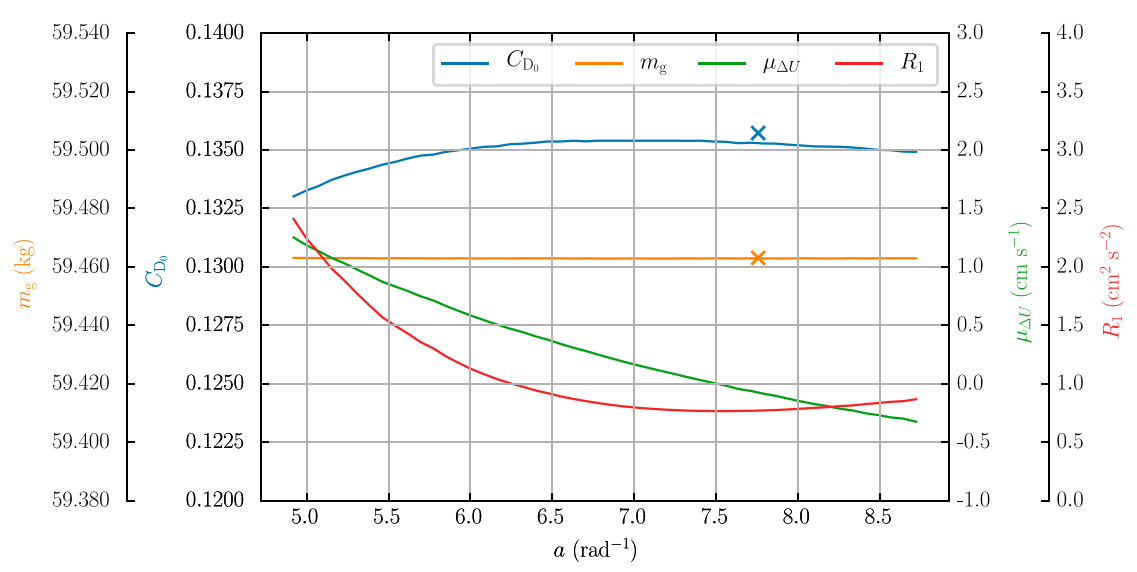

FIG. 5. Dependency of $m_{g}$ and $C_{D_{0}}$ on $a$ (left ordinate axes), and corresponding mean and standard deviations of the difference between the modeled and measured (EMC) incident velocity as well as $R_{1}$ (right ordinate axes). The cross symbols indicate the results of a minimization of $R_{2}$.

results. However, when operating gliders with microstructure sensors, the pitch battery position is usually fixed to avoid vibrations that can interfere with shear probe measurements during the moving of the pitch battery. As a consequence, especially for deep glider profiles, the pitch angle may vary substantially as a result of changes in the in situ water density and compression of the hull, so changes in flight resulting from the induced drag depend on the depth. The compressibility of the hull also causes the flight to change with depth, and hence it is difficult to distinguish between both effects.

At the end of the glider experiment in dataset II, the pitch settings of the glider COMET were varied. Over the course of a day, the target pitch was changed to three values- $\theta_{t}=16^{\circ}, 19^{\circ}, 27^{\circ}$-where the absolute values of the target pitch angles $\theta_{t}$ were the same for up- and downcasts. Using $a=7.5 \mathrm{rad}^{-1}$ as found previously, the glider flight model was calibrated for the mass and total drag coefficient, $C_{D}=C_{D_{0}}+\alpha^{2} C_{D_{1}}$, for the three subsets, each having a narrow range of pitch angles; see Fig. 6, right panel. The optimization routine yields for each pitch band a different value for $C_{D}$. Since the angle of attack can be assumed more or less constant within each pitch band, $C_{D}$ can be plotted as a function of the corresponding angle of attack; see the blue dots in Fig. 6, left panel. As the induced drag effect is proportional to the angle of attack squared, a parabola is fitted to the data, yielding $C_{D_{0}}=0.147$ and $C_{D_{1}}=10.5 \mathrm{rad}^{-2}$. The value found for the induced drag coefficient is significantly higher than the one estimated by Merckelbach et al. (2010), who suggested a total value for the induced drag of about $3 \mathrm{rad}^{-2}$. The discrepancy is most likely to be due to the protruding features that the glider has, such as the tail fin, the CTD, and, most importantly, the
Microrider package, which was not considered by Merckelbach et al. (2010).

Like the parasite drag coefficient, the induced drag coefficient is likely to change when the vehicle gets biofouled. The value quoted here was determined for a glider without noticeable biofouling. But, as argued before, the effect caused by the induced drag is of second-order importance, and some change in the induced drag coefficient caused by biofouling is likely to be insignificant.

\section{c. Results}

After calibrating the flight model for mass, parasite, and induced drag coefficients, and lift angle coefficients above, we use subsets of the data and solve both the steady-state and dynamic model to yield time series of incident water velocities. By comparing the time series with measurements, we can assess the model performance.

First, we compare the model results with measurements obtained with the DVL for two subsets of about 4 and $9 \mathrm{~h}$ of data, respectively. Measurements and model results are shown in Fig. 7 for two intervals comprising four full yos down to $40 \mathrm{~m}$ of water depth spanning about $30 \mathrm{~min}$ on 23 June 2017 (top panels) and on 24 June 2017 (bottom panels), respectively. The incident water velocity computed from the DVL measurements is available only for water depths in excess of about $10 \mathrm{~m}$ and during downcasts. The DVL measurements (blue curves, left panel) show that the glider quickly gains speed when starting the downcast and then gradually slows down as it gets deeper. This deceleration is also reproduced in the incident water velocities computed by the steady-state model (orange) and the dynamic model (red). The reason for this is that the in situ density 

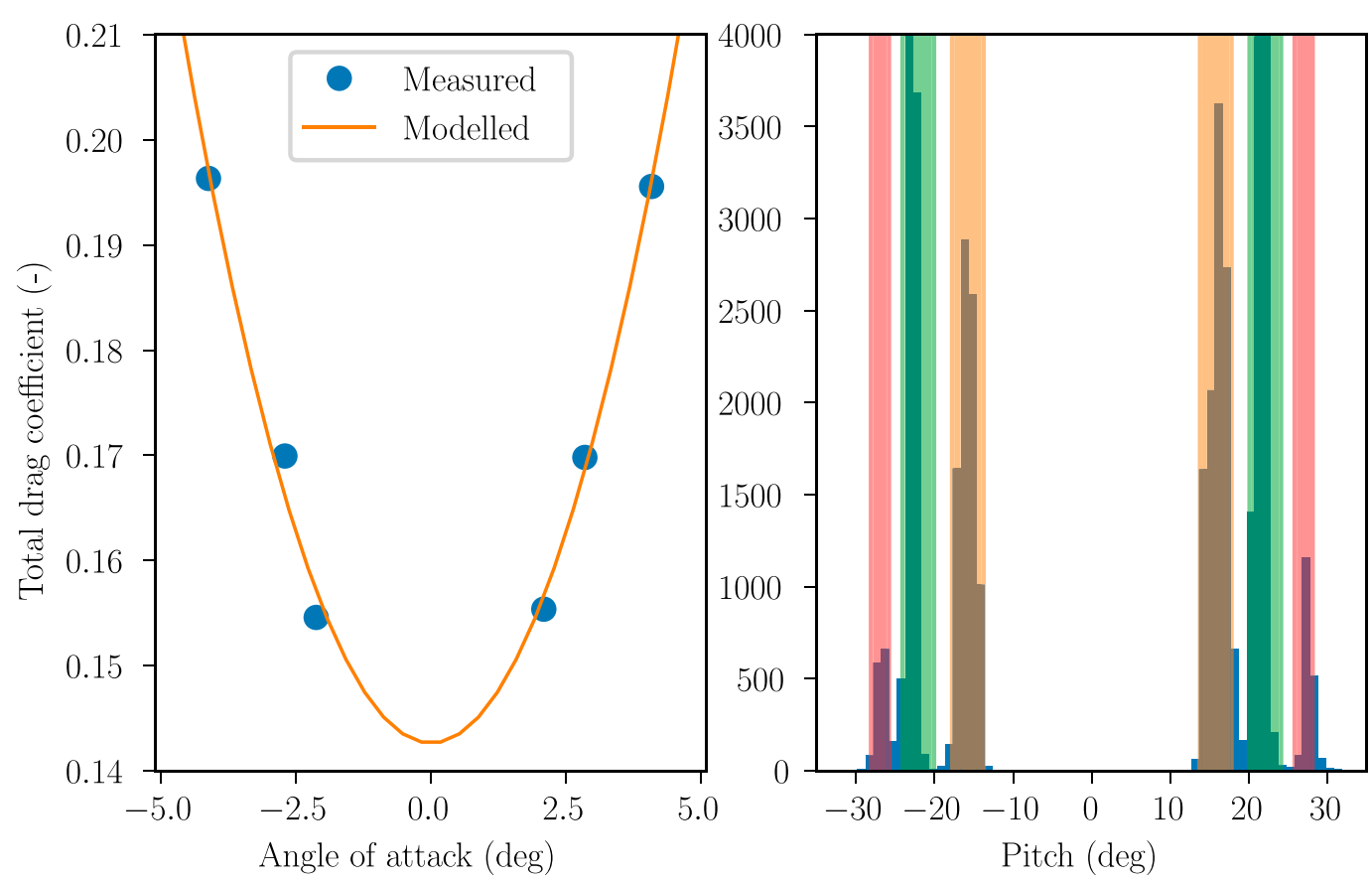

FIG. 6. (left) Total drag as a function of the angle of attack. (right) A histogram of realized pitch angles during a number of dives (dataset II). The colored bars indicate the three time subintervals selected from this dataset: gentle dives (orange), nominal dives (green), and steep dives (red).

increases with depth, reducing the glider's weight in water with depth. The deceleration observed during the upcasts is due to the same reason. In a situation like this, when the glider decelerates during most of the up- and downcasts, the dynamic model adjusts to the observed change in density with a response time on the order of a minute, whereas the steady-state model adjusts instantly. As a result, the steady-state model computes lower speeds through water than the dynamic model would for the same flight model parameter setting. So, if both models are calibrated separately, then the steady-state model has a lower drag coefficient, compensating for the lower speed through water, as we observed in section 5 (Fig. 4).

For depths greater than about $12-15 \mathrm{~m}$, down to the dive-to-climb turning points, the steady-state and dynamic models appear to yield identical results, indicating that, as expected, for most of each cast the glider's dynamical behavior can be considered steady state. Only for short periods after changing cast direction, the dynamic model shows a gradual response to the sudden changes in forcing, where the steady-state model predicts unrealistic overshoots. Discrepancies between steady-state and dynamic model near the diveto-climb turning points are present but not clearly visible in this figure. The model performance during rapid changes when changing from downcast to upcast is discussed in detail below.
Restricted to the steady-state region, both models compare favorably with the measurements. In particular for the data from 23 June (top panel), the model estimates are typically within $1-2 \mathrm{~cm} \mathrm{~s}^{-1}$ of the observations. For 24 June, however, the measurements exhibit more variability with discrepancies between observations and model estimates amounting up to about $4 \mathrm{~cm} \mathrm{~s}^{-1}$. The difference in the degree of agreement between model and observations is most likely related to environmental factors. Indeed, the variance of the water velocity relative to the bed in the flight direction, computed as an average per profile for the profiles shown, is more than 15 times larger for the profiles of 24 June than those of 23 June. Because the lift and drag force parameterizations do not account for the presence of shear, it seems likely that the increased levels of shear on 24 June contribute to the increased variability in observed incident water velocity.

A subset of data comprising close to 2 days of data of the glider IFM03 is processed in a similar way. A selection of 2 full yo cycles is shown in Fig. 8 comparing the measurements with the modeled results from the dynamic model. Since for most of the 800-m dives, the steady-state and dynamic models produce identical results, the data of the former are not displayed. Also, note that in comparison with the glider COMET, the time scale is condensed by a factor of 20 as a result of the deeper dives. 

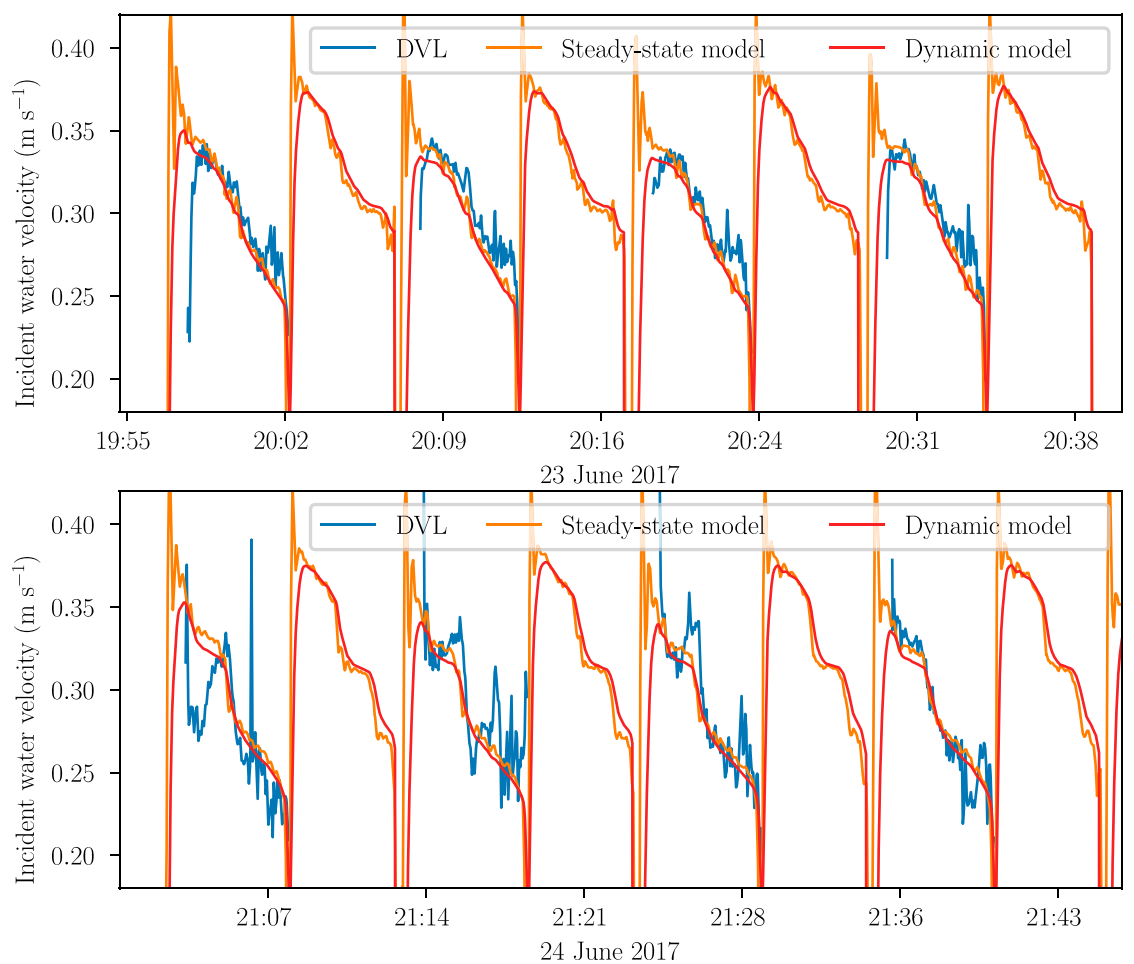

FIG. 7. A comparison of incident water velocities measured with the DVL and computed by the calibrated steady-state model and dynamic model for two half-hour intervals.

The incident water velocity measured by the EMC agrees, on average, well with the results of the dynamic model. However, some variability captured by the EMC sensor is not represented by the dynamic model. Differences between modeled and observed velocities amount to up to about $4 \mathrm{~cm} \mathrm{~s}^{-1}$ and have time scales of minutes. Like the previous dataset, most of the discrepancies between measurements and modeled results are attributed to the local variability in the flow. Another recurrent feature is that at the beginning of the first dive (and for this glider all dives, as all dive cycles within this time interval composed one full yo only), the glider accelerates slower than the model estimates. This is most likely due to trapped air bubbles, as discussed in section $6 \mathrm{c}$.

\section{Discussion \\ a. Implications for turbulent microstructure measurement}

Two key findings from the above analysis are (i) the values of calibrated drag and lift parameters are similar between two different gliders applied in different

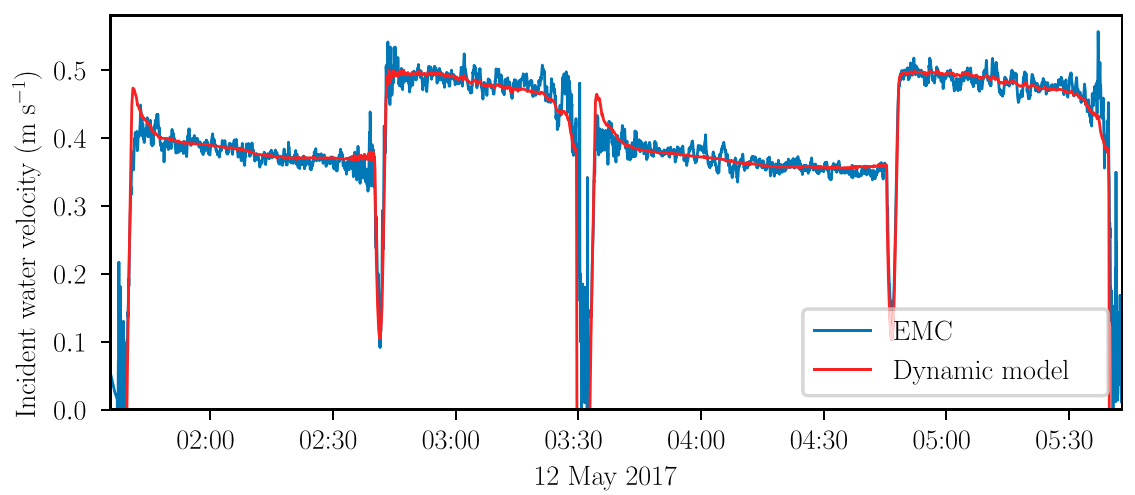

FIG. 8. A comparison of incident water velocities measured with the EMC (blue) and computed by the calibrated dynamic model (red). 
conditions, and (ii) the time series show good agreement between the observed and modeled glider speed through water $U$. A question that naturally arises is what errors a (calibrated) glider flight model then produces in $U$, and what implications this has for estimates of the dissipation rate from temperature and shear microstructure. These errors add to the uncertainty of the dissipation rate measurements over that for standard free-fall profilers, where the speed along the sensors is estimated from the pressure rate of change. Although not rigorously derived, the uncertainties of free-fall profilers are generally estimated at a factor of approximately 2 (Dewey and Crawford 1988; Moum et al. 1995).

To estimate the errors produced from deviations in the measured and modeled glider speeds, we first note the scaling of the dissipation rate $\varepsilon$ with the flow speed past the sensors, $U$. For $\varepsilon$ measured with airfoil shear probes,

$$
\varepsilon=\frac{15}{2} \nu \overline{\left(\frac{\partial v}{\partial x}\right)^{2}}
$$

where $x$ represents the distance in the glider path direction, $v$ denotes across-path velocity fluctuations, $\nu$ is the kinematic viscosity, and the bar denotes a mean. The probe returns a signal $E(t)$ that is proportional to the lift force on the probe $(U v)$, so we can express the acrosspath velocities as $v \propto E / U$. Spatial gradients of $v$ are then found using Taylor's frozen turbulence hypothesis, whereby

$$
\frac{\partial v}{\partial x}=\frac{1}{U} \frac{\partial v}{\partial t} \propto \frac{1}{U^{2}} \frac{\partial E}{\partial t}
$$

Therefore,

$$
\varepsilon \propto \frac{1}{U^{4}} \overline{\left(\frac{\partial E}{\partial t}\right)^{2}},
$$

showing that $\varepsilon$ scales with the fourth power of the flow speed past the sensors and that it will thus be sensitive to errors in its estimation. Note that if $\varepsilon$ is measured by using microstructure temperature sensors (Gregg 1999; Ruddick et al. 2000), then $\varepsilon \propto U^{-2}$ as a result of the lack of $U$ dependence arising from the lift force in the case of shear probes.

Errors in the estimation of $\varepsilon$ arising from deviations between the glider flight model and the true speed through water will therefore appear through the factor $\left(U_{\text {meas }} / U_{\text {dyn }}\right)^{n}$, where $n=\{2,4\}$ for measurements from microstructure temperature sensors and shear probes, respectively, and the $U$ ratio corresponds to the measured speed to that obtained from the dynamic model.
These factors are computed as profile averages (indicated by angled braces) in Fig. 9 from the DVL datasets. It shows averages as solid lines with the shaded area indicating twice the standard deviation in the data for both DVL datasets. Depths shallower than $12 \mathrm{~m}$ are not shown, since no reliable DVL data can be collected (see also section 4c). Although some bias toward positive or negative differences-amounting to close to $1 \mathrm{~cm} \mathrm{~s}^{-1}$ exists, the bias is not systematic, as it is different for two consecutive deployments. When multiple deployments are considered, there is negligible bias in the difference between mean measured and modeled speeds.

The histograms in Fig. 9 show that $90 \%$ of the errors expected in dissipation rate estimates as a result of modeled glider speeds are within a factor of $0.67-1.43$ for shear probe measurements $(n=4)$ and within 0.82 1.26 for temperature microstructure $(n=2)$. These errors should be compared to the factor-of-2 uncertainty normally associated with dissipation rate measurements from vertical profilers (Dewey and Crawford 1988; Moum et al. 1995). In addition, it is not clear how much of this difference between modeled and measured speeds can be attributed to the need to use DVL measurements that are not coincident in time and space with the glider position, and require averaging to reduce measurement noise.

An advantage the EMC sensor has over the DVL is that it measures velocities collocated in time and space with the glider flight model. As long as the instantaneous angle of attack remains small, the measured quantity represents the glider speed through water, an assumption we have made in the analysis. The same profile averaged quantities as in Fig. 9, but now for the EMC sensor, are shown in Fig. 10. For depths deeper than $150 \mathrm{~m}$, we also find unbiased results, with error ratios that have a smaller spread than for the DVL [i.e., $90 \%$ of the data lie in the ranges of $0.83<\left\langle\left(U_{\mathrm{EMC}} / U_{\mathrm{dyn}}\right)^{4}<1.20\right.$ and $0.91<\left\langle\left(U_{\mathrm{EMC}} / U_{\mathrm{dyn}}\right)^{2}<1.09\right]$. The velocity measurements made by the EMC sensor have a standard deviation that is about an order of magnitude smaller than the readings from the DVL (section 4a), so this suggests that a fraction of the spread of the data observed for the DVL (and possibly also for the EMC) could be due to uncertainty in the measurements.

If the steady-state model were applied to the present data but using a lift angle coefficient of $6.1 \mathrm{rad}^{-1}$ (Merckelbach et al. 2010), then the bias in the difference between measured and modeled incident velocities would be 1.3 and $0.5 \mathrm{~cm} \mathrm{~s}^{-1}$ for datasets I (Fig. 4) and III (Fig. 5), respectively. The associated biases in the estimates for the dissipation rate applied to dataset I (dataset III) would an underestimation of a factor of 1.10 (1.05) and 1.20 (1.07) for estimates from 

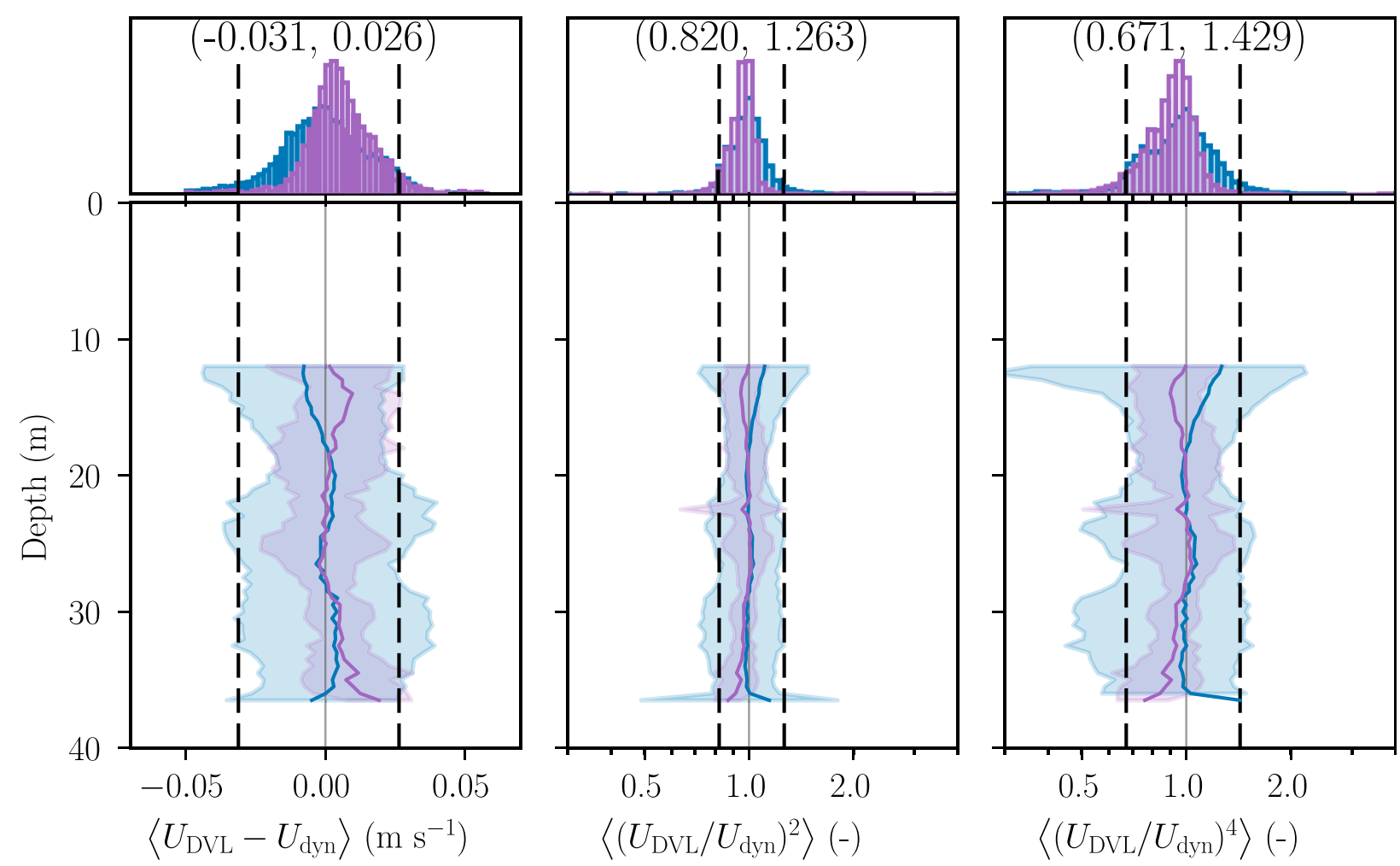

FIG. 9. A comparison of the glider speed through water, computed from DVL measurements and the dynamic flight model (downcasts only). The panels show profile averages of the difference between (left) the velocities, (middle) the squared ratio of the velocities, and (right) the ratio of the velocity raised to the fourth power (solid lines). The shaded region in the corresponding color indicates the spread in the data, computed from twice the standard deviation. The histograms above the profiles show the distribution of the corresponding observations. The data from a 4-h deployment period (1751-2158 UTC 23 Jun 2017; purple), and the data from 9-h deployment period (1754 UTC 24 Jun 2017-0304 UTC 25 Jun 2017; blue). The 5 and 95 percentiles, computed over both datasets, are marked (dashed lines).

temperature microstructure sensors and shear probes, respectively. The bias sensitivity to the error in the specification of the lift angle coefficient depends on the steepness of the dives and climbs, where smaller pitch angles cause larger biases.

\section{b. Dynamic versus steady-state flight}

Thus far we have concluded that for most of the dive and climb, the glider can be considered in a regime of steady state. This is demonstrated by the close agreements between steady-state and dynamic model results throughout most of the flight (Figs. 7 and 8). Since implementing the dynamic model is computationally more expensive, this brings up the question of whether the extra effort is needed. The dynamic model better handles situations that involve sudden and significant changes in the forcing. Examples of such instances are when pitch battery positions change during flight, when the glider experiences a strong pycnocline, or during the transit from downcast to upcast. Standard practice is to discard microstructure data when steady-state conditions are not met. Employing a dynamic model, however, may somewhat relax this restriction.

To examine this in more detail, we focus on a transition from dive to climb. Figure 11 is a zoomed-in view of such an event of the time series from Fig. 8, showing the speed through water during the last segment of a downcast, its transition to the upcast (marked by the gray box) and a full upcast, until the glider reached the surface. Most of the flight is in the steady-state regime, as the results of the steady-state model (orange curve) and dynamic model (red curve) are indiscernible in this regime. During the transition from downcast to upcast (gray box, and highlighted in the inset), the model results diverge and the flight is not steady state. As soon as the glider initiates the transition, the pitch battery is set to its position for the upcast, suddenly increasing the pitch angle (green curve in the inset). Concurrently, the measured speed through water is seen to decrease. After reaching a minimum velocity of $12 \mathrm{~cm} \mathrm{~s}^{-1}$, the glider gradually accelerates, until the flight becomes steady state again. The steady-state model computes 

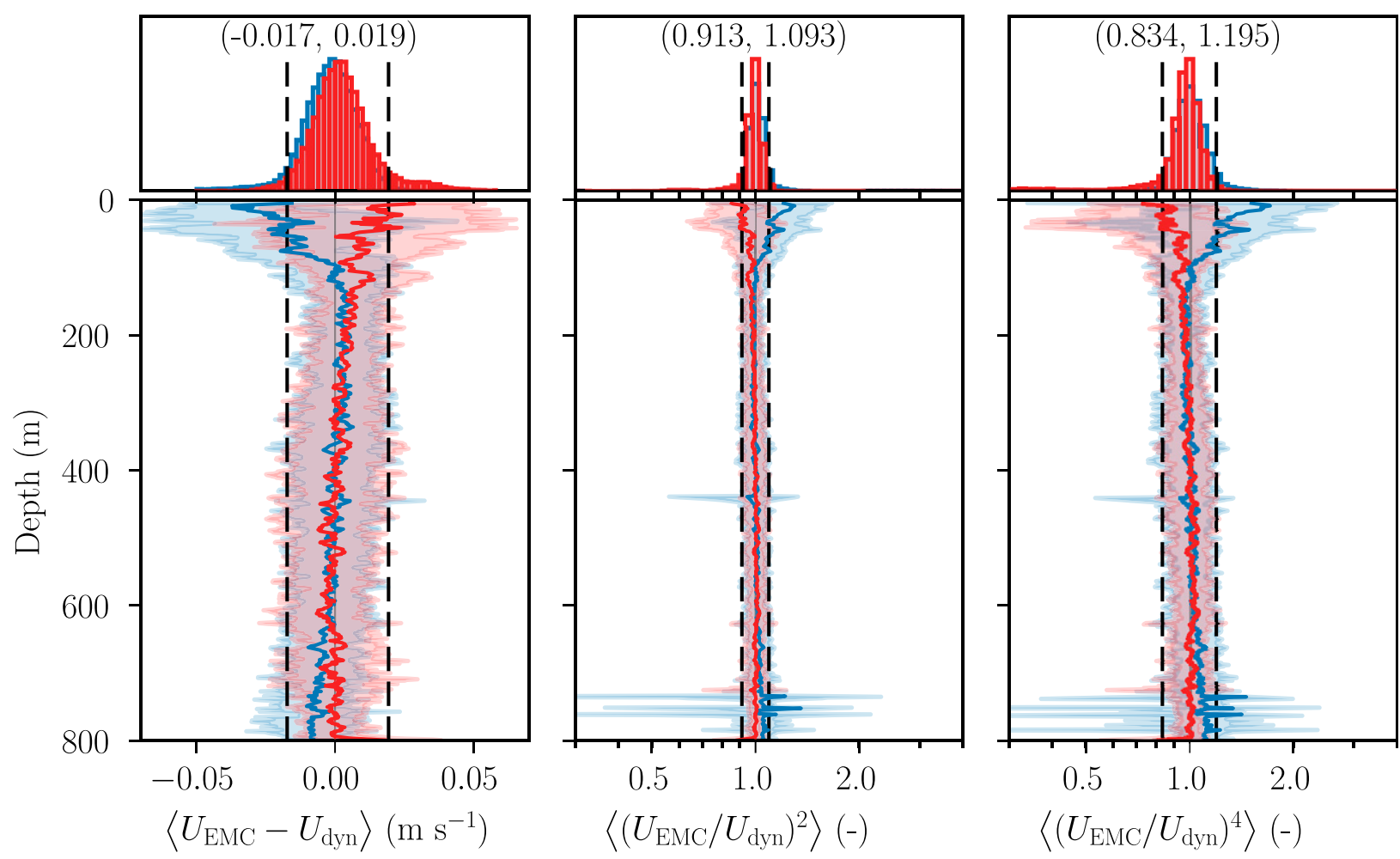

FIG. 10. A comparison of the glider speed through water, computed from EMC measurements and the dynamic flight model. The panels show profile averages of the difference between (left) the velocities, (middle) the squared ratio of the velocities, and (right) the ratio of the velocity raised to the fourth power (solid lines). The shaded region in the corresponding color indicates the spread in the data, computed from twice the standard deviation. The downcasts (blue) and upcasts (red) are indicated. The histograms above the profiles show the distribution of the corresponding observations. The 5 and 95 percentiles, computed over both the down- and upcasts combined, are marked (dashed lines). The data encompass a 45-h period (0020 UTC 12 May-2113 UTC 13 May 2017).

false behavior during the transition. The dynamic model, however, does reproduce the dip in speed.

\section{c. Flight model error sources}

Although errors in the velocity measurements lead to discrepancies between measured and modeled incident velocities, some of these discrepancies can be related to the model failing to capture all aspects of the glider flight. For water depths less than $150 \mathrm{~m}$, discrepancies between observed and modeled velocities are very clearly present in the dataset of glider IFM03 (Fig. 10). It is unlikely that these discrepancies can be related to inaccurate velocity measurements. It is, however, a known issue for Slocum gliders with an oil-based displacement pump, such as the glider IFM03, that air can diffuse into the oil system. Air bubbles in the oil system will lead to inaccurate reporting of the actual glider volume change, causing the flight model to compute erroneous buoyancy forces and as a result erroneous flight velocities. Because there is currently no way to measure how much air is present, and where it resides, this aspect is not included in the flight model. The effect of air bubbles can be identified by comparing the computed vertical glider velocities with the observed depth rate; if calibrated for deep dives, the presence of air bubbles manifests itself as a bias in the vertical velocity difference during the shallower part of the dives.

A further assumption underlying both the steadystate and dynamic flight models is that the ocean currents are steady and free of shear. The consequence is that in the presence of, for example, vertical shear and internal waves with short periods, the inertia of the glider can cause the instantaneous incident velocity to be significantly different from the modeled incident velocity, introducing errors in the drag and lift forces computed by the model. Unfortunately, this issue is not remedied by the dynamic model developed herein, despite having the inertial terms included. This is because the model cannot discern between the glider velocity in an inertial frame, the time derivative of which equals the acceleration, and the incident water velocity (relative to the glider), which is used in the parameterizations of the lift and drag forces. The results shown in Figs. 7 and 8 indicate that the discrepancies observed between the 


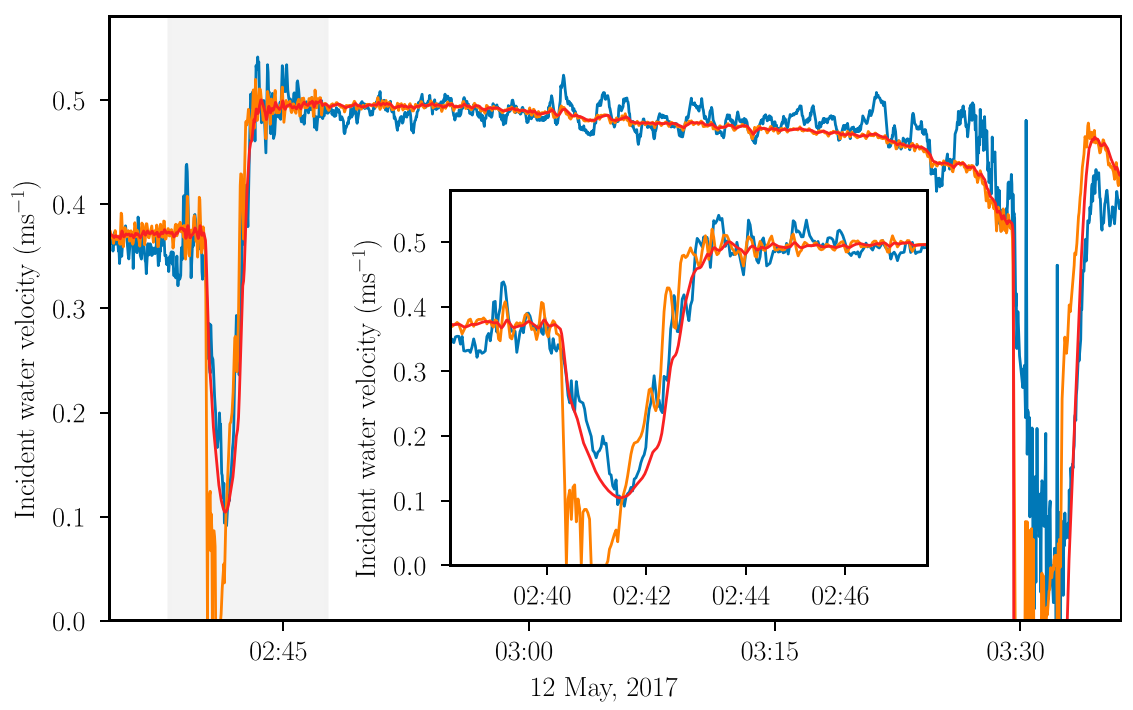

FIG. 11. A section of a time series showing the speed through water as measured by the EMC (blue curve), the steady-state model (orange), and a dynamic glider flight model (red). The inset is a zoomed-in view of a deep transition from dive to climb.

measured incident velocities and the dynamic model results occur on time scales on the order of $1-10 \mathrm{~min}$, and are likely caused by the model failing to capture the effects caused by an unsteady ocean.

The present measurements suggest that the steady ocean assumption introduces errors in the estimates of the dissipation rate that are within acceptable levels given the current uncertainties in microstructure estimates. This may not be true anymore for more dynamic environments than those encountered in this study, so in those conditions measuring the dissipation rate with an acceptable accuracy may still require direct measurements of the glider flight.

\section{Conclusions}

This study is the first to use measurements of in situ glider flight to test, calibrate, and extend a glider flight model. Our principal motivation is to quantify and reduce uncertainties in the use of flight models for turbulent microstructure studies. Calibration of the steadystate model of Merckelbach et al. (2010) resulted in changes in the lift angle coefficient to $a=7.5 \mathrm{rad}^{-1}$ as well as the induced drag coefficient $C_{D_{1}}=10.5 \mathrm{rad}^{-1}$. This change in the value of the lift angle coefficient from that reported in Merckelbach et al. (2010) results in a reduction of the difference between measured and modeled incident water velocities of about $0.5-1.3 \mathrm{~cm} \mathrm{~s}^{-1}$.

Measurements of in situ glider flight allow us to quantify errors in dissipation rate calculations associated with errors in the incident water velocity predicted by the flight model. Using velocities from the EMC as a baseline, we found that
$90 \%$ of the estimates of the dissipation rate based on the calibrated flight model are within a factor of 1.1 and 1.2 for measurements derived from microstructure temperature sensors and shear probes, respectively. The uncalibrated model would produce a bias of factors of $1.05-1.10$ and $1.07-1.20$ for temperature and shear microstructure, respectively. The uncertainty in dissipation rate estimates can be attributed to the local variability in the flow, which is not accounted for in the flight model, as well as possible noise from the velocity measurement itself. When using water velocities measured with the DVL, for which the estimates are more prone to instrument noise, the factors are slightly larger, namely, 1.2 and 1.4, respectively.

To better represent the hydrodynamics, we have extended the steady-state glider flight model of Merckelbach et al. (2010) to a dynamic model by including the inertial and added mass terms. A comparison of the two models found that the flight is largely well described by the steady-state model and that only when conditions change rapidly, such as during dive-climb transitions, does the steady-state model fail, whereas the dynamic model predicts the incident water velocity reasonably well.

Acknowledgments. We are grateful to Rolf Lueck (Rockland Scientific) for the useful discussion on the interpretation of the velocities measured with the electromagnetic current meter. We also thank David Pheifer (True North Technologies) for sharing his expertise on how to correct the attitude data of glider COMET. The bathymetry data used in this work were provided by GEBCO (www.gebco.net). This paper is a contribution to the projects M6 and T2 of the Collaborative Research 
Centre TRR 181, "Energy Transfer in Atmosphere and Ocean"; and the project B6 of the Collaborative Research Centre SFB 754, "Climate-Biogeochemistry Interactions in the Tropical Ocean"- -all funded by the German Research Foundation (Deutsche Forschungsgemeinschaft). Part of the measurements (dataset I) took place during the Expedition Clockwork Ocean, which was partially supported by the Helmholtz-Zentrum Geesthacht as part of its PACES II program. We thank the captain and crew of the R/V Elisabeth Mann Borgese for the excellent technical support, and express our appreciation for the constructive comments and suggestions by two anonymous reviewers. The data used in this manuscript are available online (https://doi.org/10.5281/zenodo.2270123).

\section{REFERENCES}

Anderson, B. D. O., and J. B. Moore, 2005: Optimal Filtering. Dover Books on Electrical Engineering, Dover Publications, 368 pp.

Dewey, R., and W. Crawford, 1988: Bottom stress estimates from vertical dissipation rate profiles on the continental shelf. J. Phys. Oceanogr., 18, 1167-1177, https://doi.org/10.1175/ 1520-0485(1988)018<1167:BSEFVD>2.0.CO;2.

Fer, I., A. K. Peterson, and J. E. Ullgren, 2014: Microstructure measurements from and underwater glider in the turbulent Faroe Bank Channel overflow. J. Atmos. Oceanic Technol., 31, 1128-1150, https://doi.org/10.1175/JTECH-D-13-00221.1.

Garau, B., S. Ruiz, W. G. Zhang, A. Pascual, E. Heslop, J. Kerfoot, and J. Tintoré, 2011: Thermal lag correction on Slocum CTD glider data. J. Atmos. Oceanic Technol., 28, 1065-1071, https:// doi.org/10.1175/JTECH-D-10-05030.1.

Gregg, M., 1999: Uncertainties and limitations in measuring $\varepsilon$ and $\chi_{T}$.J. Atmos. Oceanic Technol., 16, 1483-1490, https://doi.org/ 10.1175/1520-0426(1999)016<1483:UALIMA>2.0.CO;2.

Griffiths, G., Ed., 2002: Technology and Applications of Autonomous Underwater Vehicles. Ocean Science and Technology Series, CRC Press, 368 pp., https://doi.org/10.1201/9780203522301.

Imlay, F. H., 1961: The complete expressions for "added mass" of a rigid body moving in an ideal fluid. David Taylor Model Basin Research and Development Rep. 1528, 31 pp.

Merckelbach, L., 2018a: A glider flight model for Slocum ocean gliders. GitHub, https://github.com/smerckel/gliderflight/tree/1.0.1.

_ 2018b: Initial release of glider flight model. Version 1.0.1, Zenodo, https://doi.org/10.5281/zenodo.2222694.

— D. Smeed, and G. Griffiths, 2010: Vertical velocities from underwater gliders. J. Oceanic Atmos. Technol., 27, 547-563, https://doi.org/10.1175/2009JTECHO710.1.

Moum, J., M. Gregg, R. Lien, and M. Carr, 1995: Comparison of turbulence kinetic energy dissipation rate estimates from two ocean microstructure profilers. J. Atmos. Oceanic Technol., 12, 346-366, https://doi.org/10.1175/1520-0426(1995)012<0346: COTKED $>2.0 . \mathrm{CO} ; 2$.

Newman, J. N., 1977: Marine Hydrodynamics. MIT Press, 402 pp., https://doi.org/10.7551/mitpress/4443.001.0001.

Palmer, M., G. Stephenson, M. Inall, C. Balfour, A. Düsterhus, and J. Green, 2015: Turbulence and mixing by internal waves in the Celtic Sea determined from ocean glider microstructure measurements. J. Mar. Syst., 144, 57-69, https://doi.org/10.1016/ j.jmarsys.2014.11.005.

Peterson, A. K., and I. Fer, 2014: Dissipation measurements using temperature microstructure from an underwater glider. Methods Oceanogr., 10, 44-69, https://doi.org/10.1016/j.mio.2014.05.002.

Rockland Scientific, 2017: MicroPod-EM: Electromagnetic flow sensor. Rockland Scientific Rep., 2 pp., https://rocklandscientific. com/wp-content/uploads/2017/05/RSI-Data-Sheet-MicroPodEMA4-1_00-web.pdf.

Ruddick, B., A. Anis, and K. Thompson, 2000: Maximum likelihood spectral fitting: The Batchelor spectrum. J. Atmos. Oceanic Technol., 17, 1541-1555, https://doi.org/10.1175/15200426(2000)017<1541:MLSFTB > 2.0.CO;2.

Rudnick, D., 2016: Ocean research enabled by underwater gliders. Annu. Rev. Mar. Sci., 8, 519-541, https://doi.org/10.1146/ annurev-marine-122414-033913.

Scheifele, B., S. Waterman, L. Merckelbach, and J. Carpenter, 2018: Measuring the dissipation rate of turbulent kinetic energy in strongly stratified, low-energy environments: A case study from the Arctic Ocean. J. Geophys. Res. Oceans, 123, 5459-5480. https://doi.org/10.1029/2017JC013731.

Schultze, L. K. P., L. M. Merckelbach, and J. R. Carpenter, 2017: Turbulence and mixing in a shallow stratified shelf sea from underwater gliders. J. Geophys. Res. Oceans, 122, 9092-9109, https://doi.org/10.1002/2017JC012872.

Simon, D., 2006: Optimal State Estimation: Kalman, $H^{\infty}$, and Nonlinear Approaches. Wiley and Sons, 526 pp.

St. Laurent, L., and S. Merrifield, 2017: Measurements of near-surface turbulence and mixing from autonomous ocean gliders. Oceanography, 30 (2), 116-125, https://doi.org/10.5670/oceanog.2017.231.

Teledyne RD Instruments, 2017: Explorer Doppler velocity log (DVL): Navigation performance in a compact package. Teledyne RD Instruments Rep., 2 pp., http://www.teledynemarine.com/ Lists/Downloads/explorer_datasheet_hr.pdf.

Todd, R., D. Rudnick, J. Sherman, W. Owens, and L. George, 2017: Absolute velocity estimates from autonomous underwater gliders equipped with Doppler current profilers. J. Atmos. Oceanic Technol., 34, 309-330, https://doi.org/10.1175/ JTECH-D-16-0156.1.

Wolk, F., R. Lueck, and L. St. Laurent, 2009: Turbulence measurements from a glider. Proc. 13th Workshop on Physical Processes in Natural Waters, Palermo, Italy, University of Palermo, https://rocklandscientific.com/wp-content/uploads/ 2015/01/Wolk_Lueck_StLaurent_final_PPNW.pdf. 